PROCEEDINGS OF THE

AMERICAN MATHEMATICAL SOCIETY

Volume 137, Number 2, February 2009, Pages 615-625

S 0002-9939(08)09510-5

Article electronically published on August 26, 2008

\title{
ON FOURIER RESTRICTION AND THE NEWTON POLYGON
}

\author{
ÁKOS MAGYAR \\ (Communicated by Andreas Seeger)
}

\begin{abstract}
Local $L^{p} \rightarrow L^{2}$ bounds are proved for the restriction of the Fourier transform to analytic surfaces of the form $S=(x, f(x))$ in $\mathbb{R}^{3}$. It is found that the range of exponents is determined by the so-called distance of the Newton polygon, associated to $f$, except when the principal quasi-homogeneous part of $f(x)$ contains a factor of high multiplicity. The proofs are based on the method of Phong-Stein and Rychkov, adapted to scalar oscillatory integrals.
\end{abstract}

\section{INTRODUCTION}

If $S \subset \mathbb{R}^{n+1}$ is a compact piece of a smooth hypersurface, a central question of harmonic analysis is to determine the pair of exponents $p, q \geq 1$ for which the Fourier restriction operator $\left.\phi \rightarrow \widehat{\phi}\right|_{S}$ maps $L^{p}\left(\mathbb{R}^{n+1}\right) \rightarrow L^{q}(S)$.

This problem is still open even in the case of the unit sphere $S^{2} \subset \mathbb{R}^{3}$. However the case $q=2$ for surfaces of everywhere nonvanishing curvature was answered by the classic works of Stein and Tomas; see [S], T].

Note that the restriction property is local by nature, and is invariant under affine maps $x \rightarrow A x+b$ (where $\operatorname{det} A \neq 0$ ). Thus for real analytic $S$, it is enough to study the local problem, where $S=\{(x, f(x)): x \in V\}, V \subseteq \mathbb{R}^{n}$ is an open neighborhood of the origin and $f: V \rightarrow \mathbb{R}$ is a real analytic function such that $f(0)=\nabla f(0)=0$. We say that the Fourier restriction property $R_{S}(p \rightarrow q)$ holds at the origin if there exists a neighborhood $U$ of 0 so that for all $C^{\infty}$-functions $\psi$ with compact support in $U$, the inequality

$$
\left(\int_{U}|\widehat{\phi}(\xi, f(\xi))|^{q} \psi(\xi) d \xi\right)^{1 / q} \leq C_{p, q, f, \psi}\|\phi\|_{L^{p}\left(\mathbb{R}^{n+1}\right)}
$$

holds. The aim of this paper is to draw attention to the relation between the range of exponents $p, q$ for which the restriction property $R_{S}(p \rightarrow q)$ holds and a numeric invariant, the so-called distance of the Newton polygon of the function $f$.

If $f(x)=\sum_{k \in \mathbb{Z}_{+}^{n}} a_{k} x^{k}$ is the Taylor expansion of $f(x)$, then its Newton polyhedron is defined by

$$
N_{f}=\operatorname{Conv}\left(\bigcup_{k \in A} k+\mathbb{R}_{+}^{n}\right) \text {, }
$$

Received by the editors August 20, 2007, and, in revised form, January 25, 2008 2000 Mathematics Subject Classification. Primary 42B10; Secondary 43A32.

Key words and phrases. Fourier transform, oscillatory integrals, Newton polygon.

This research was supported in part by NSF Grant DMS-0456490. 
where $A=\left\{k: a_{k} \neq 0\right\}$ is the support of the sequence $\left\{a_{k}\right\}$. The smallest positive number $d=d_{f}$ such that the point $\mathbf{d}=(d, \ldots, d)$ is in $N_{f}$ is called the distance of $N_{f}$ and its reciprocal $\delta_{f}=1 / d_{f}$ the Newton decay rate. If the point $\mathbf{d}_{f}=\left(d_{f}, \ldots, d_{f}\right)$ lies on a compact edge $\alpha$, then $\alpha$ is called the principal edge of $N_{f}$.

First we give the following necessary condition.

Theorem 1. Let $S=(x, f(x))$ where $f: V \rightarrow \mathbb{R}$ is an analytic function such that $f(0)=\nabla f(0)=0$, and let $d \sigma=\psi d x$ be a measure on $S$ in local coordinates $x$ where $\psi$ is a smooth function of small support.

If the local $R_{S}(p \rightarrow q)$ restriction property holds at the origin, then one has

$$
p^{\prime} \geq q\left(1+d_{f}\right) \quad \text { where } \frac{1}{p}+\frac{1}{p^{\prime}}=1
$$

and $d_{f}$ is the distance of the Newton polyhedron $N_{f}$.

The idea of the proof of (1.2) is doing the well-known Knapp example, in the general case of an analytic hypersurface, in other words, to test the restriction property (1.1) on functions $\phi_{\delta}$ whose Fourier transform $\widehat{\phi}_{\delta}$ is supported on a box $B_{\delta}$ best fitted to the surface $S$.

However the Knapp example is not always sharp; a simple example is the graph of the function: $f(x, y)=\left(y-x^{2}\right)^{m}$ for $m>3$ (another example is described in Sh] $)$. Indeed, if one tests the restriction property $R_{S}(p \rightarrow q)$ on the function $\phi_{\delta}$ whose Fourier transform $\widehat{\phi}_{\delta}$ is the characteristic function of the box $B_{\delta}=\{|x| \leq$ $\left.1,|y| \leq 1,|z| \leq \delta^{m}\right\}$, then it is easy to see that

$$
\left(\int_{U}\left|\widehat{\phi}_{\delta}(x, y, f(x, y))\right|^{q} \psi(x, y) d x d y\right)^{1 / q} \geq C_{q, \psi} \delta^{\frac{1}{q}},
$$

while $\left\|\phi_{\delta}\right\|_{L^{p}\left(\mathbb{R}^{3}\right)} \leq C_{p} \delta^{\frac{m}{p^{\prime}}}$ for all $0<\delta<1$. Thus the restriction property only holds for exponents $p, q$ satisfying: $p^{\prime} \geq q m$, which is more restrictive than the one obtained from the Knapp example: $p^{\prime} \geq q(1+2 m / 3)$, given in (1.2), as $d_{f}=2 m / 3$ for the function $f(x, y)=\left(y-x^{2}\right)^{m}$.

Thus one needs additional conditions on the function $f$. One such condition is the so-called $\mathbb{R}$-nondegeneracy of Varchenko; see $[\mathrm{AV}$. However, as we shall see, that may be too restrictive, especially when the surface has curvatures vanishing to a high order, that is, when $d_{f}$ is large. Our partial result of sufficiency is taking this into consideration.

Let $n=2$ and let $\alpha$ be a compact edge of the boundary of the Newton polygon $N_{f}$. Define the quasi-homogeneous part of $f$ by corresponding to $\alpha$ by

$$
f_{\alpha}(x, y)=x^{-A^{\prime}} y^{-B} \sum_{(k, l) \in \alpha} a_{k l} x^{k} y^{l}
$$

if $\alpha$ is connecting the points $(A, B)$ and $\left(A^{\prime}, B^{\prime}\right)$ where $A^{\prime}<A$ and $B<B^{\prime}$. We define a number $r_{\alpha}$, called the multiplicity of $f_{\alpha}$.

Definition 1. If $\alpha$ is a compact edge, then the multiplicity $r_{\alpha}$ of $f_{\alpha}$ is defined to be the minimum of the highest multiplicity of a real root of $\partial_{y} f_{\alpha}(1, y)$ and of the highest multiplicity of a real root of $\partial_{x} f_{\alpha}(x, 1)$. If $\alpha$ is an infinite edge, then we set $r_{\alpha}=0$ 
If $\alpha$ is the principal edge of $N_{f}$, then $f_{\alpha}$ is called the principal part of $f$ and we write $r_{f}$ for $r_{\alpha}$. By the above definition, if the point $\mathbf{d}_{\mathbf{f}}$ is on an infinite edge, then $r_{f}=0$.

Theorem 2. Let $S=(x, y, f(x, y))$, where $f: \mathbb{R}^{2} \rightarrow \mathbb{R}$ is an analytic function such that $f(0,0)=\nabla f(0,0)=0$. Then the local $R_{S}(p \rightarrow 2)$ restriction property holds at $(0,0)$ for

$$
p^{\prime}>2\left(1+\max \left(d_{f}, r_{f}+1\right)\right) .
$$

Note that in case $r_{f}<d_{f}$, (1.2) and (1.4) give the sharp range of exponents $p$ (up to the endpoint) for which the local $R_{S}(p \rightarrow 2)$ restriction property holds. This includes functions with factors of high multiplicity, which are $\mathbb{R}$-degenerate in the sense of Varchenko (which means that the principal part of $f$ has distinct real factors; see below). For example take $f(x, y)=\left(y-a x^{2}\right)^{m}\left(y-b x^{2}\right)^{m}(0<a<b)$ for any $m \geq 2$; indeed in this case $r_{f}=m-1$ and $d_{f}=\frac{4}{3} m$.

The proof of (1.4) is based on an oscillatory integral estimate.

Theorem 3. Let the function $f(x, y)$ be an analytic function such that $f(0,0)=$ $\nabla f(0,0)=0$. Then for a smooth cut-off function $\psi(x, y)$ of sufficiently small support, one has the bound for the associated oscillatory integral

$$
|I(\lambda)|=\left|\int_{\mathbb{R}^{2}} e^{i \lambda f(x, y)} \psi(x, y) d x d y\right| \leq C_{\varepsilon}(1+|\lambda|)^{-\min \left(\delta_{f}, \frac{1}{r_{f}+1}\right)+\varepsilon} \forall \varepsilon>0 .
$$

This compares to the result of Varchenko in dimension 2, which shows $|I(\lambda)| \leq$ $C_{\varepsilon}(1+\lambda)^{-\delta_{f}+\varepsilon}$ under the so-called $\mathbb{R}$-nondegeneracy condition. The function $f$ is called $\mathbb{R}$-nondegenerate if

$$
\partial_{x} f_{\alpha}(x, y)=\partial_{y} f_{\alpha}(x, y)=0 \quad \text { implies } \quad x=0 \quad \text { or } \quad y=0,
$$

where $f_{\alpha}$ is the principal part of $f$ defined in (1.3). This condition however only holds when neither $\partial_{x} f_{\alpha}(x, 1)$ nor $\partial_{y} f_{\alpha}(1, y)$ has multiple real roots, and in particular only when $r_{f}=1$. Indeed, assuming that there is a nonzero $x_{0} \in \mathbb{R}$ such that

$$
f_{\alpha}\left(x_{0}, 1\right)=\partial_{x} f_{\alpha}\left(x_{0}, 1\right)=0
$$

and using the Euler relations

$$
f_{\alpha}\left(x_{0}, 1\right)=\frac{1}{m} \partial_{x} f_{\alpha}\left(x_{0}, 1\right)+\frac{1}{n} \partial_{y} f_{\alpha}\left(x_{0}, 1\right), \quad \text { where } m=A-A^{\prime}, \quad n=B^{\prime}-B,
$$

we see that $\partial_{y} f_{\alpha}\left(x_{0}, 1\right)=0$ and hence $f$ is $\mathbb{R}$-degenerate.

The proof of (1.5) exploits a factorization, called the Puiseux product, as was done similarly for oscillatory integral operators in [PS1] and [R].

The route from (1.5) to (1.4) is standard, but based on a highly nontrivial result of Karpushkin (see [K], PS3]), namely the local stability of the decay rate of oscillatory integrals with analytic phase in 2 dimensions. In fact it implies that for $\xi=\lambda(-1, u, v)$ the estimate

$$
|\widehat{d \sigma}(\xi)|=\left|\int_{\mathbb{R}^{2}} e^{i \lambda(f(x, y)-u x-v y)} \psi(x, y) d x d y\right| \leq C_{\varepsilon}(1+|\lambda|)^{-\min \left(\delta_{f}, \frac{1}{r_{f}+1}\right)+\varepsilon}
$$

for all $\varepsilon>0$ holds uniformly for $(u, v)$ being in a small neighborhood of $(0,0)$, and then also for all $(u, v)$, since outside the neighborhood the gradient of the phase is large: $\left|\left(\partial_{x} f-u, \partial_{y} f-v\right)\right| \geq C \lambda$. Thus the integral is rapidly and uniformly 
decreasing. Let us remark that one of the main obstacles to proving similar results in higher dimensions is the lack of the stability theorem.

Finally, by the classical argument of [T], a uniform bound of the form $|\widehat{d \sigma}(\xi)| \leq$ $C(1+|\xi|)^{-\beta}$ implies that the local $R_{S}(p \rightarrow 2)$ restriction property holds for $p^{\prime}>$ $2\left(1+\beta^{-1}\right)$ (in fact, by Greenleaf's theorem [G], the restriction property also holds for $p^{\prime} \geq 2\left(1+\beta^{-1}\right)$ ), which gives (1.4). Thus Theorem 2 follows from Theorem 3 .

\section{NeCESSARY CONDITIONS}

In this section we prove Theorem 1. Let $f: \mathbb{R}^{n} \rightarrow \mathbb{R}$ be an analytic function such that $f(0,0)=\nabla f(0,0)=0$, and let $\Gamma_{f}$ be the boundary and $V_{f}$ be the set of vertices of its Newton polyhedron. We use the notation $|x|^{a}=\left|x_{1}\right|^{a_{1}}\left|x_{2}\right|^{a_{2}} \ldots\left|x_{n}\right|^{a_{n}}$ for $x \in \mathbb{R}^{n}$ and $a=\left(a_{1}, a_{2}, \ldots, a_{n}\right) \in \mathbb{Z}^{n}$.

Lemma 1. One has for $x$ being in a sufficiently small neighborhood of the origin:

$$
|f(x)| \leq C \max _{b \in V_{f}}|x|^{b} .
$$

Proof. Let us introduce the ordering on $\mathbb{Z}_{+}^{n}: a \prec b$ if $b \in a+\mathbb{Z}_{+}^{n}, b \neq a$; and set of minimal elements: $A^{\prime}=\{a \in A: \nexists b \in A, b \prec a\}$. It is easy to see by an induction on $n$ that $A^{\prime}$ is finite; thus $V_{f}$ is finite as well. Since the Taylor series of $f(x)$ converges, one has for every $a \in A^{\prime}$,

$$
\sum_{k \in A, a \preceq k}\left|a_{k}\right||x|^{k} \leq C_{a}|x|^{a} .
$$

It follows that

$$
|f(x)| \leq C \max _{a \in A^{\prime}}|x|^{a} .
$$

Moreover for every $a \in A^{\prime}$ there are vertices $b_{1}, \ldots, b_{n}$ (not necessarily distinct) such that for a convex combination of them: $b=\sum_{i} \lambda_{i} b_{i} \preceq a$. Indeed every point on the boundary $\Gamma_{f}$ is of this form. Thus

$$
|x|^{a} \leq \prod_{i}|x|^{\lambda_{i} b_{i}} \leq \max _{i}|x|^{b_{i}} .
$$

The lemma follows from (2.2).

Definition 2. We say that $a \in \mathbb{R}_{+}^{n}$ is admissible if

$$
k \cdot a \geq 1 \text { for every } k \in \Gamma_{f} .
$$

Lemma 2. Let

$$
\delta=\min \left\{\sum_{j=1}^{n} a_{j}: a=\left(a_{1}, \ldots, a_{n}\right) \text { is admissible }\right\} .
$$

Then $\delta=\delta_{f}=1 / d_{f}$.

Proof. If $a$ is admissible, then $a \cdot \mathbf{d}_{\mathbf{f}}=d_{f} \sum_{j} a_{j} \geq 1$; hence $\delta \geq \delta_{f}$. On the other hand, let $\alpha$ be the face of $N_{f}$ containing $\mathbf{d}_{f}$ and let $a \in \mathbb{R}^{n}$ be defined such that $a \cdot k=1$ for every $k \in \alpha$. Then by the convexity of $N_{f}$ the plane through $\alpha$ separates 0 and $N_{f}$; thus $a \cdot l \geq 1$ for every $l \in N_{f}$. In particular $a \in \mathbb{R}_{+}^{n}$ and $a$ is admissible. Also $a \cdot \mathbf{d}_{\mathbf{f}}=d_{f} \sum_{j} a_{j}=1$ and thus $\delta \leq \delta_{f}$. 
Proof of Theorem 1. Let $\psi(x)$ be a smooth cut-off function of small support and let $a=\left(a_{1}, \ldots, a_{n}\right)$ be admissible. For $0 \leq \tau<1$ define the function $\phi_{a, \tau}$ such that

$$
\widehat{\phi}_{a, \tau}\left(x_{1}, \ldots, x_{n+1}\right)=\psi\left(\tau^{-a_{1}} x_{1}, \ldots, \tau^{-a_{n}} x_{n}, \tau^{-1} x_{n+1}\right) .
$$

If $\left|x_{j}\right| \leq \tau^{a_{j}}$ for $1 \leq j \leq n$, then since $a$ is admissible, one has by (2.1),

$$
|f(x)| \leq C \max _{k \in V_{f}} \tau^{k \cdot a} \leq C \tau,
$$

and thus (choosing $\psi$ appropriately), $\left|\widehat{\phi}_{a, \tau}(x, f(x))\right| \geq c>0$. Hence

$$
\int_{S}\left|\widehat{\phi}_{a, \tau}\right|^{q} d \sigma=\int_{\mathbb{R}^{n}}\left|\widehat{\phi}_{a, \tau}(x, f(x))\right|^{q} \psi(x) d x \geq c \tau^{\nu_{a}},
$$

where $\nu_{a}=\sum_{j} a_{j}$. On the other hand, by scaling, one has that $\left\|\phi_{a, \tau}\right\|_{p} \approx \tau^{\left(1+\nu_{a}\right) / p^{\prime}}$.

If the $R_{S}(p \rightarrow q)$ restriction property holds, then one must have

$$
\tau^{\nu_{a} / q} \leq C \tau^{\left(1+\nu_{a}\right) / p^{\prime}}
$$

for every $0<\tau<1$ and admissible $a$. Thus by Proposition 2,

$$
p^{\prime} \geq \max _{\text {a admissible }} q\left(1+\nu_{a}^{-1}\right)=q\left(1+d_{f}\right) .
$$

This proves Theorem 1 .

\section{SUfFICIENT CONDITIONS}

We will need the following standard Van der Corput type lemma for oscillatory integrals with polynomial-type phases, proved in [PS2]; see (3), p. 42 in the proof of Theorem 1 there.

Lemma 3. Let $I=[a, b]$ be an interval of length at most $1, \chi \in \mathcal{C}^{1}(\mathbb{R})$ be a smooth function and let $f$ be a real and $\mathcal{C}^{2}(\mathbb{R})$. Assume that $\left|f^{\prime}(y)\right| \geq \delta>0$ for all $y \in I$ and $f^{\prime \prime}(y)$ has at most $d$ roots in $I$. Then one has for $\lambda>0$,

$$
\left|\int_{I} e^{i \lambda f(y)} \chi(y) d y\right| \leq \lambda^{-1} \delta^{-1}(2 d+1)\left(\sup _{I}|\chi|+\sup _{I}\left|\chi^{\prime}\right|\right) .
$$

We remark that inequality (3.1) remains true, with $d=0$, when $f^{\prime \prime}(y)$ is constant 0 on the interval $I$, which is easy to see by performing an integration by parts and using that $f^{\prime}(y)$ is constant on $I$.

To apply the above lemma for a function of two variables $F(x, y)$, we invoke an immediate corollary of the Weierstrass Preparation Theorem.

Lemma 4. Let $U \subseteq \mathbb{R}^{2}$ be an open neighborhood of $(0,0)$, and let $F: U \rightarrow \mathbb{R}$ be a nonzero analytic function. Then there is an $\eta>0$ and a positive integer $d$ such that for every $0<|x|<\eta$, the function $y \rightarrow F(x, y)$ has at most $d$ roots in $[-\eta, \eta]$.

Proof. Let $K=\min \left\{k: a_{k l} \neq 0\right\}$, where $F(x, y)=\sum_{k, l} a_{k l} x^{k} y^{l}$ and write $F(x, y)$ $=x^{K} G(x, y)$. The function $G(0, y)$ is not identically zero. Thus by the Weierstrass Preparation Theorem (see $[\mathrm{H}]$ Sec. 7.5)

$$
G(x, y)=U(x, y)\left(c_{d} y^{d}+c_{d-1}(x) y^{d-1}+\ldots+c_{0}(x)\right),
$$

where $c_{d} \neq 0$, and $U(x, y) \neq 0$ for $|x| \leq \eta,|y| \leq \eta, \eta>0$ being sufficiently small. Thus for any $|x| \leq \eta$, the function $y \rightarrow F(x, y)$ can have at most $d$ roots. 
We describe the Newton polygon $N_{F}$ associated to an analytic function $F$ in more detail. If $\alpha$ runs through the compact edges of the boundary $\Gamma_{F}$, connecting the vertices $\left(A_{\alpha}, B_{\alpha}\right)$ and $\left(A_{\alpha}^{\prime}, B_{\alpha}^{\prime}\right)$ where $A_{\alpha}>A_{\alpha}^{\prime}$, then put $n_{\alpha}=B_{\alpha}^{\prime}-B_{\alpha}$ and $\gamma_{\alpha}=\frac{A_{\alpha}-A_{\alpha}^{\prime}}{B_{\alpha}^{\prime}-B_{\alpha}}$. Also let $A$ be the $x$ and $B$ be the $y$ coordinate of the vertical and horizontal infinite edges of $\Gamma_{F}$. The intersection of the bisector and line of the edge $\alpha$ is the point $\left(d_{\alpha}, d_{\alpha}\right)$, where $d_{\alpha}=\left(A_{\alpha}+\gamma_{\alpha} B_{\alpha}\right) /\left(1+\gamma_{\alpha}\right)$. By convexity, $d_{f} \geq d_{\alpha}$ for all compact edges $\alpha$. Thus writing $\delta_{\alpha}=1 / d_{\alpha}=\left(1+\gamma_{\alpha}\right) /\left(A_{\alpha}+\gamma_{\alpha} B_{\alpha}\right)$, one finds that

$$
\delta_{f}=\min _{\alpha}\left(1 / A, 1 / B, \delta_{\alpha}\right) .
$$

We introduce the ordering $\alpha \prec \beta$ if $\gamma_{\alpha}<\gamma_{\beta}$ and note that

$$
A_{\alpha}=A+\sum_{\beta \preceq \alpha} n_{\beta} \gamma_{\beta} \quad \text { and } \quad B_{\alpha}=B+\sum_{\beta \succ \alpha} n_{\beta} .
$$

Also, $N_{F}$ has some symmetry properties: if $G(x, y)=F( \pm x, \pm y)$, then $N_{F}=N_{G}$; if $G(x, y)=F(y, x)$, then $N_{G}$ is obtained by reflecting $N_{F}$ to the bisector $y=x$. The germ of an analytic function $F(x, y)$ admits a factorization called the Puiseux product (see $[\mathrm{R}]$ ) of the form

$$
F(x, y)=U(x, y) x^{A} y^{B} \prod_{\alpha} \prod_{i=1}^{n_{\alpha}}\left(y-y_{\alpha i}(x)\right),
$$

where $U(x, y)$ and $c_{\alpha i}$ are nonzero, and $\left.y_{\alpha i}(x)\right)$ is asymptotic to a fractional power series of the form $c_{\alpha i} x^{\gamma_{\alpha}}+c_{\alpha^{\prime} i} x^{\gamma_{\alpha}+\gamma}+\ldots$, as $x \rightarrow 0$. In particular, for any given $\tau>0$ one has

$$
\left|y_{\alpha i}(x)-c_{\alpha i} x^{\gamma_{\alpha}}\right| \leq \tau x^{\gamma_{\alpha}}
$$

for $|x|<c \tau^{1 / \gamma}, c>0$ being a small constant depending only on $F$. Moreover there is a fixed large constant $D>0$ such that if $2^{-j-1} \leq x<2^{-j}$ and $2^{-k-1} \leq y<2^{-k}$ for $j, k \geq J$ large enough, then

$$
\left|y-y_{\alpha i}(x)\right| \sim\left\{\begin{array}{lll}
2^{-j \gamma_{\alpha}} & \text { if } \quad k \geq j \gamma_{\alpha}+D \\
2^{-k} & \text { if } \quad k \leq j \gamma_{\alpha}-D
\end{array}\right.
$$

where $x \sim y$ means that $C^{-1} x \leq y \leq C x$ for some constant $C>0$ whose value depends only on $F$ and can change from place to place. By the notation $k \gg j \gamma_{\alpha}$ it is meant that $k-j \gamma_{\alpha} \geq D$. We remark that both conditions can be achieved by choosing the support of $\psi(x, y)$ small enough.

One may use the Puiseux product to estimate the size of $F(x, y)$ on the dyadic rectangles $R_{j k}=\left[2^{-j-1}, 2^{-j}\right] \times\left[2^{-k-1}, 2^{-k}\right]$ which are "away" from the zero set of F.

Lemma 5. Let $F(x, y)$ be an analytic function, such that $F(0,0)=0$. Then one has the following size estimates:

(i) Let $\alpha \prec \alpha^{\prime}$ be two consecutive compact edges of the Newton polygon $N_{F}$, and let $\left(A_{\alpha}, B_{\alpha}\right)$ denote the common vertex of the edges $\alpha$ and $\alpha^{\prime}$. If $j \gamma_{\alpha} \ll k \ll \gamma_{\alpha^{\prime}}$, then for $(x, y) \in R_{j k}$,

$$
|F(x, y)| \sim 2^{-j A_{\alpha}-k B_{\alpha}} .
$$

(ii) Let $\alpha_{0}$ denote the minimal edge of $N_{F}$ (i.e. $\gamma_{\alpha_{0}} \leq \gamma_{\alpha} \forall \alpha$ ), and let $\left(A_{0}, B_{0}\right)$ denote the common vertex of the edge $\alpha_{0}$ and the infinite vertical edge. If $k \ll j \gamma_{\alpha_{0}}$, 
then for $(x, y) \in R_{j k}$,

$$
|F(x, y)| \sim 2^{-j A_{0}-k B_{0}} .
$$

(iii) Let $\alpha_{s}$ denote the maximal edge of $N_{F}$ (i.e. $\gamma_{\alpha_{s}} \geq \gamma_{\alpha} \forall \alpha$ ), and let $\left(A_{s}, B_{s}\right)$ denote the common vertex of the edges $\alpha_{s}$ and the infinite horizontal edge. If $k \gg j \gamma_{\alpha_{s}}$, then for $(x, y) \in R_{j k}$,

$$
|F(x, y)| \sim 2^{-j A_{s}-k B_{s}} .
$$

Proof. Assume first $j \gamma_{\alpha} \ll k \ll \gamma_{\alpha^{\prime}}$. Then by (3.6) for $(x, y) \in R_{j k}$ one has $\left|y-y_{\beta i}(x)\right| \sim 2^{-j \gamma_{\beta}}$ for $\beta \preceq \alpha$ and $\left|y-y_{\beta i}(x)\right| \sim 2^{-k}$ for $\beta \succ \alpha$. Thus by (3.3) and (3.4),

$$
|F(x, y)| \sim 2^{-j\left(A+\sum_{\beta \preceq \alpha} n_{\beta} \gamma_{\beta}\right)} 2^{-k\left(B+\sum_{\beta \succ \alpha} n_{\beta}\right)}=2^{-j A_{\alpha}-k B_{\alpha}} .
$$

The proof of estimates (3.7) and (3.8) proceeds the same way, noting that $A_{0}=A$ and $B_{s}=B$.

In the proof of Theorem 3, we will apply the above size estimates to either $F=\partial_{x} f$ or $F=\partial_{y} f$. Thus we will need some information on the Newton polygons associated to $\partial_{x} f$ and $\partial_{y} f$, which we will denote by $N_{x}$ and $N_{y}$. Note that $N_{x}$ is obtained from $N_{f}$ by shifting it to the left by 1 and maybe replacing the minimal edge $\alpha_{0}$ by an edge $\alpha_{0}^{\prime}$, a series of edges $\alpha_{0}^{\prime} \succ \ldots \succ \alpha_{-r}^{\prime}$, or an infinite edge. These replacements can only happen when the infinite vertical edge of $N_{f}$ is the $y$-axis. An important observation here is that, by convexity, $\gamma_{\alpha_{0}^{\prime}} \geq \gamma_{\alpha_{0}}$. The analysis of $N_{y}$ is similar: here $N_{f}$ is shifted down by 1 , and the maximal edge $\alpha_{s}$ may be replaced by edges $\alpha_{s}^{\prime} \prec \ldots \prec \alpha_{t}^{\prime}$ such that $\gamma_{\alpha_{s}} \leq \gamma_{\alpha_{s}^{\prime}}$, or the horizontal infinite edge.

Proof of Theorem 3. Following $[\mathrm{R}$, one decomposes the support of the integral in (1.5) into the four quadrants of $\mathbb{R}^{2}$ and notes that each of the resulting integrals are treated in exactly the same way because the Newton polygon is invariant w.r.t. coordinate changes: $x \leftrightarrow-x, y \leftrightarrow-y$. Thus we assume that the integration is taking place for $x>0, y>0$ and define

$$
I_{j k}(\lambda)=\int_{R_{j k}} e^{i \lambda f} \psi
$$

Since only those rectangles $R_{j k}$ intersecting the support of $\psi$ contribute to the integral, one may assume $j, k \geq J$ for a sufficiently large $J$.

We start with the (rather) special case when $N_{f}$ has no compact edges. Then the Puiseux product takes the form

$$
f(x, y)=U(x, y) x^{A} y^{B},
$$

where $A>0$ or $B>0$ by our assumption on $f$. Assume $B>0$ and let $F=\partial_{y} f$. For $(x, y) \in R_{j k}$ one has

$$
|F(x, y)| \sim x^{A} y^{B-1} \sim 2^{-j A-k(B-1)} .
$$

Thus applying Lemma 3 for fixed $2^{-j-1} \leq x \leq 2^{-j}$ and integrating in $x$, one obtains

$$
\left|I_{j k}(\lambda)\right| \leq C \lambda^{-1} 2^{j(A-1)+k(B-1)} .
$$

Taking the geometric mean of this and the size estimate $\left|I_{j k}\right| \leq 2^{-j-k-2}$ gives

$$
\left|I_{j k}(\lambda)\right| \leq C \lambda^{-\delta} 2^{j(\delta A-1)+k(\delta B-1)}
$$


for any $0 \leq \delta \leq 1$. Choosing $\delta=\delta_{f}-\varepsilon$, where $\delta_{f}=\min \left(\frac{1}{A}, \frac{1}{B}\right)$ and $\varepsilon>0$, one has

$$
|I(\lambda)| \leq \sum_{j, k \geq J}\left|I_{j k}(\lambda)\right| \leq C_{\varepsilon} \lambda^{-\delta_{f}+\varepsilon}
$$

for any $\varepsilon>0$. The case $A>0$ is handled analogously, choosing $F=\partial_{x} f$.

Now assume that $N_{f}$ has at least one compact edge, and for a fixed compact edge $\alpha$ define

$$
I_{\alpha}(\lambda)=\sum_{j \gamma_{\alpha} \ll k \ll j \gamma_{\alpha^{\prime}}} I_{j k}(\lambda) \text { and } I^{\alpha}(\lambda)=\sum_{\left|j \gamma_{\alpha}-k\right|<D} I_{j k}(\lambda),
$$

where $\alpha \prec \alpha^{\prime}$ are consecutive edges. Moreover let

$$
I^{-}(\lambda)=\sum_{k \ll j \gamma_{\alpha_{0}}} I_{j k}(\lambda) \text { and } I^{+}(\lambda)=\sum_{k \gg j \gamma_{\alpha_{s}}} I_{j k}(\lambda) .
$$

Next, we estimate the quantities $I^{ \pm}(\lambda)$. Let $\alpha_{0}$ be the minimal edge of $N_{f}$ connecting the points $\left(A_{0}, B_{0}\right)$ and $\left(A^{\prime}, B^{\prime}\right)$ with $B_{0}>B^{\prime}$. Then $\left(A_{0}, B_{0}-1\right)$ is the highest vertex point of the Newton polygon $N_{y}$. We argue that $\gamma_{\alpha_{0}} \leq \gamma_{\alpha_{0}^{\prime}}$, where $\alpha_{0}^{\prime}$ denotes the minimal edge of $N_{y}$. Indeed, if $B^{\prime}>0$, then $\alpha_{0}^{\prime}$ is parallel to $\alpha_{0}$. Hence $\gamma_{\alpha_{0}} \leq \gamma_{\alpha_{0}^{\prime}}$; otherwise $N_{f}$ has only one compact edge. Hence $\alpha_{0}=\alpha_{s}$ and the estimate follows from our previous analysis of $N_{y}$. Thus $k \ll j \gamma_{\alpha_{0}} \leq j \gamma_{\alpha_{0}^{\prime}}$ and (3.7) applies for $F(x, y)=\partial_{y} f(x, y)$ giving

$$
|F(x, y)| \sim 2^{-j A_{0}-k\left(B_{0}-1\right)},
$$

similarly as in (3.10). Proceeding as in (3.11)-(3.13) one obtains for $0 \leq \delta \leq 1$,

$$
\left|I_{j k}(\lambda)\right| \leq C \lambda^{-\delta} 2^{j\left(\delta A_{0}-1\right)+k\left(\delta B_{0}-1\right)}=2^{k\left(\delta B_{0}+A_{0} / \gamma_{\alpha_{0}}\right)-\left(1+1 / \gamma_{\alpha_{0}}\right)} 2^{r\left(\delta A_{0}-1\right)},
$$

where the last equality was obtained by writing $j=k / \gamma_{\alpha_{0}}+r$. By our assumption $r \geq D^{\prime}>0, j \geq J$; thus choosing $\delta=\min \left(\delta_{\alpha_{0}}, 1 / A_{0}\right)-\varepsilon \geq \delta_{f}-\varepsilon$, one gets

$$
\left|I^{-}(\lambda)\right| \leq \sum_{j \geq J, r \geq D^{\prime}}\left|I_{j k}(\lambda)\right| \leq C_{\varepsilon} \lambda^{-\delta_{f}+\varepsilon} .
$$

The quantity $I^{+}(\lambda)$ is estimated analogously by choosing $F=\partial_{x} f$ and $\delta=$ $\min \left(\delta_{\alpha_{s}}, 1 / B_{s}\right)-\varepsilon$.

To estimate $I_{\alpha}(\lambda)$ let $\alpha \prec \alpha^{\prime}$ be consecutive edges of $N_{f}$ with common vertex $\left(A_{\alpha}, B_{\alpha}\right)$ and let $j \gamma_{\alpha} \ll k \ll j \gamma_{\alpha^{\prime}}$. First assume that $A_{\alpha} \geq B_{\alpha}>0$ and note that $d_{f} \geq B_{\alpha}$ by the convexity of $N_{f}$. The point $\left(A_{\alpha}, B_{\alpha}-1\right)$ is the common vertex of two edges $\beta \prec \beta^{\prime}$ of the Newton polygon $N_{y}$ (where $\beta^{\prime}$ may be infinite), and by our previous analysis, $\gamma_{\beta}=\gamma_{\alpha}$ and $\gamma_{\alpha^{\prime}} \leq \gamma_{\beta^{\prime}}$ (taking $\gamma_{\beta^{\prime}}=+\infty$ if $\beta^{\prime}$ is infinite). Thus $j \gamma_{\beta} \ll k \ll j \gamma_{\beta^{\prime}}$, and estimate (3.7) (or (3.9)) applies to $F(x, y)=\partial_{y} f(x, y)$ when $(x, y) \in R_{j k}$, which gives $|F(x, y)| \sim 2^{-j A_{\alpha}-k\left(B_{\alpha}-1\right)}$. Proceeding as before and substituting $k=j \gamma_{\alpha}+r$, one has

$$
\left|I_{j k}(\lambda)\right| \leq C \lambda^{-\delta} 2^{j\left(\delta\left(A_{\alpha}+\gamma_{\alpha} B_{\alpha}\right)-\left(1+\gamma_{\alpha}\right)\right)} 2^{r\left(\delta B_{\alpha}-1\right)} .
$$

Choosing $\delta=\min \left(\delta_{\alpha}, 1 / B_{\alpha}\right)-\varepsilon \geq \delta_{f}-\varepsilon$ and summing in $j \geq J, r \geq D$, one gets

$$
\left|I_{\alpha}(\lambda)\right| \leq C_{\varepsilon} \lambda^{-\delta_{f}+\varepsilon}
$$

The case $B_{\alpha} \geq A_{\alpha}$ is handled analogously, choosing $F=\partial_{x} f, \delta=\min \left(\delta_{\alpha^{\prime}}, 1 / A_{\alpha}\right)-$ $\varepsilon \geq \delta_{f}-\varepsilon$ and substituting $j=k / \gamma_{\alpha^{\prime}}+r$. 
The multiplicity condition enters in the estimates for $I^{\alpha}(\lambda)$, that is, when $\left|k-j \gamma_{\alpha}\right| \leq D$. Consider first a nonprincipal edge $\alpha$ of $N_{f}$. Then $\alpha$ lies completely below or above the bisector $y=x$. Assume the first case, that is, when $A_{\alpha}^{\prime}>B_{\alpha}^{\prime}>0$. Then the point $\left(A_{\alpha}^{\prime}, B_{\alpha}^{\prime}-1\right)$ is a vertex of $N_{y}$. Let $\alpha^{\prime}$ be the edge of $N_{y}$ with upper vertex $\left(A_{\alpha}^{\prime}, B_{\alpha}^{\prime}-1\right)$; then $\gamma_{\alpha^{\prime}} \geq \gamma_{\alpha}$. If $\gamma_{\alpha^{\prime}}>\gamma_{\alpha}$, then $k \ll j \gamma_{\alpha^{\prime}}$, and our analysis reduces to the cases $\left(I_{\alpha}(\lambda)\right.$ or $\left.I^{-}(\lambda)\right)$ considered earlier. In fact, it is easier here as one needs to sum only for $j \geq J$ using the fact that $k-j \gamma_{\alpha}$ is bounded. One obtains

$$
\left|I^{\alpha}(\lambda)\right| \leq C \sum_{j \geq J}\left|I_{j k}(\lambda)\right| \leq C_{\varepsilon} \lambda^{-\delta_{\alpha}+\varepsilon} .
$$

So we will assume that $\gamma_{\alpha^{\prime}}=\gamma_{\alpha}$ from now on.

Let $F(x, y)=\partial_{y} f(x, y)$ and consider its Puiseux product defined in (3.4). It is clear that the product

$$
F_{\alpha^{\prime}}(x, y):=x^{A_{\alpha^{\prime}}^{\prime}} y^{B_{\alpha^{\prime}}} \prod_{i=1}^{n_{\alpha^{\prime}}}\left(y-c_{\alpha^{\prime} i} x^{\gamma_{\alpha}}\right)
$$

is a quasi-homogenous polynomial whose support lies on the edge $\alpha^{\prime}$. It is wellknown, and is not hard to see, that $F_{\alpha^{\prime}}(x, y)$ is in fact the quasi-homogeneous part of $F(x, y)$ corresponding to $\alpha^{\prime}$ defined in (1.3). Moreover, since $\gamma_{\alpha^{\prime}}=\gamma_{\alpha}$, a point $(k, l)$ is on $\alpha^{\prime}$ if and only if the point $(k, l+1)$ is on $\alpha$ and hence $F_{\alpha^{\prime}}=\left(\partial_{y} f\right)_{\alpha^{\prime}}=\partial_{y}\left(f_{\alpha}\right)$.

Let $\left\{c_{1}, \ldots, c_{t}\right\}$ be the set of the real coefficients $c_{\alpha^{\prime} i}$, and write

$$
\partial_{y} f_{\alpha}(x, y)=F_{\alpha^{\prime}}(x, y)=x^{A_{\alpha^{\prime}}^{\prime}} y^{B_{\alpha^{\prime}}} \prod_{l=1}^{t}\left(y-c_{l} x^{\gamma_{\alpha}}\right)^{r_{l}} \prod_{i: c_{\alpha^{\prime} i}}\left(y-c_{\alpha^{\prime} i} x^{\gamma_{\alpha}}\right)
$$

and let $r_{\alpha}=\max _{l} r_{l}$. By the above remarks it is easy to see that $r_{\alpha}$ is the highest multiplicity of a real root of $\partial_{y} f_{\alpha}(1, y)$.

For each $1 \leq l \leq t$ and for each $x$, define the clusters: $\mathcal{C}_{x, l}=\left\{\operatorname{Re} y_{\alpha i}(x): c_{\alpha i}=\right.$ $\left.c_{l}\right\}$, where $R e z$ denotes the real part of $z$. Observe that by (3.5) the diameter of each cluster is at most $\tau 2^{-j \gamma_{\alpha}}$, while the distance between them is at least $C 2^{-j \gamma_{\alpha}}$, where $\tau>0$ can be chosen sufficiently small.

For a fixed $x$ we make use of the Whitney decomposition of the set $\left[2^{-k-1}, 2^{-k}\right] /$ $\mathcal{C}_{x}$, where $\mathcal{C}_{x}=\left\{\operatorname{Re} y_{\alpha i}(x): c_{\alpha^{\prime} i}\right.$ is real $\}$. It is a collection of intervals $I_{m}$ of length $\sim 2^{-m}$ such that the distance of a point $y \in I_{m}$ from the set $C_{x}$ is again $\sim 2^{-m}$. Clearly, one can assume $m \geq j \gamma_{\alpha}$ and for a given $m$ there are at most $2 n_{\alpha^{\prime}}$ of the intervals $I_{m}$. If $\mathcal{C}_{l}$ is the closest cluster to $I_{m}$, then for $y \in I_{m}$ one has

$$
\left|y-y_{\alpha^{\prime} i}(x)\right| \geq \begin{cases}C 2^{-m} & \text { if } c_{\alpha^{\prime} i} \text { is real, and } y_{\alpha^{\prime} i}(x) \in \mathcal{C}_{l}, \\ C 2^{-j \gamma_{\alpha}} & \text { otherwise }\end{cases}
$$

Note that the intervals $I_{m}$ may depend on $x$ but the above estimates are uniform in $x \in\left[2^{-j-1}, 2^{-j}\right]$.

Thus one estimates the size of $F(x, y)$ for $y \in I_{m}$ :

$$
\begin{gathered}
|F(x, y)| \geq C 2^{-(j A+k B)} \prod_{\beta \prec \alpha^{\prime}} 2^{-j n_{\beta} \gamma_{\beta}} \prod_{\beta \succ \alpha^{\prime}} 2^{-k n_{\beta}} 2^{-m r_{l}} 2^{-\left(n_{\alpha^{\prime}}-r_{l}\right) j \gamma_{\alpha}} \\
\geq C 2^{-j\left(A_{\alpha}^{\prime}+\gamma_{\alpha}\left(B_{\alpha}^{\prime}-1\right)\right)} 2^{r_{\alpha}\left(m-j \gamma_{\alpha}\right)}
\end{gathered}
$$


using the facts that $2^{-k} \sim 2^{-j \gamma_{\alpha}}, r_{\alpha} \geq r_{l}, m \geq j \gamma_{\alpha}$ and that $\left(A_{\alpha}^{\prime}, B_{\alpha}^{\prime}-1\right)$ is the upper vertex of $\alpha^{\prime}$. Next, one uses Lemma 3, which yields

$$
\left|I_{j k, m}(x, \lambda)\right|:=\left|\int_{I_{m}} e^{i \lambda f(x, y)} \psi(x, y) d y\right| \leq C \lambda^{-1} 2^{j A_{\alpha}^{\prime}+k\left(B_{\alpha}^{\prime}-1\right)} 2^{r_{\alpha}\left(m-j \gamma_{\alpha}\right)}
$$

uniformly for $x \in\left[2^{-j-1}, 2^{-j}\right]$.

As in the previous case, this can be balanced against the trivial estimate $\left|I_{j k, m}\right| \leq$ $C 2^{-m}$ to obtain that for any $\varepsilon>0$,

$$
\left|I_{j k, m}(x, \lambda)\right| \leq C \lambda^{-\frac{1}{r_{\alpha}+1}+\varepsilon} 2^{-m \varepsilon} 2^{j \frac{A_{\alpha}^{\prime}+\gamma_{\alpha}\left(B_{\alpha}^{\prime}-1\right)-r_{\alpha} \gamma_{\alpha}}{r_{\alpha}+1}} 2^{-\varepsilon j\left(A_{\alpha}^{\prime}+\gamma_{\alpha}\left(B_{\alpha}^{\prime}-1\right)-r_{\alpha} \gamma_{\alpha}\right) .}
$$

Summing in $m$ and integrating in $x$ give

$$
\left|I_{j k}(\lambda)\right| \leq C_{\varepsilon} \lambda^{-\frac{1}{r_{\alpha}+1}+\varepsilon} 2^{j\left(\frac{A_{\alpha}^{\prime}+\gamma_{\alpha}\left(B_{\alpha}^{\prime}-1\right)-r_{\alpha} \gamma_{\alpha}}{r_{\alpha}+1}-1\right)} 2^{-\varepsilon j\left(A_{\alpha}^{\prime}+\gamma_{\alpha}\left(B_{\alpha}^{\prime}-1\right)-r_{\alpha} \gamma_{\alpha}\right)} .
$$

Notice that the exponent $\frac{A_{\alpha}^{\prime}+\gamma_{\alpha}\left(B_{\alpha}^{\prime}-1\right)-r_{\alpha} \gamma_{\alpha}}{r_{\alpha}+1}-1$ is nonnegative if and only if

$$
r_{\alpha}+1 \leq\left(A_{\alpha}^{\prime}+\gamma_{\alpha} B_{\alpha}^{\prime}\right) /\left(1+\gamma_{\alpha}\right)=d_{\alpha} .
$$

In this case, choosing $\delta=\left(r_{\alpha}+1\right) \delta_{\alpha}-2 \varepsilon$ to balance against the trivial estimate $\left|I_{j k}(\lambda)\right| \leq C 2^{-j\left(1+\gamma_{\alpha}\right)}$ to kill the $j$ factors, a straightforward computation shows

$$
\left|I^{\alpha}(\lambda)\right| \leq \sum_{\left|k-j \gamma_{\alpha}\right| \leq D}\left|I_{j k}(\lambda)\right| \leq C_{\varepsilon} \sum_{\left|k-j \gamma_{\alpha}\right| \leq D} 2^{-j \varepsilon} \lambda^{-\delta_{\alpha}+\varepsilon} \leq C_{\varepsilon} \lambda^{-\delta_{\alpha}+\varepsilon} .
$$

We argue that the multiplicity condition (3.29) holds when $\alpha$ is a nonprincipal edge lying under the bisector $y=x$. Indeed, then $r_{\alpha}+1 \leq n_{\alpha^{\prime}}+1 \leq B_{\alpha^{\prime}}^{\prime}+1=$ $B_{\alpha}^{\prime}<d_{\alpha}$.

If $\alpha$ is a nonprincipal edge lying above the bisector, then one has to repeat the above analysis by reversing the roles of $x$ and $y$. More formally, let $\tilde{f}(x, y):=$ $f(y, x)$. Note that $I_{j k}(\lambda, f)=I_{k j}(\lambda, \tilde{f})$ and $N_{\tilde{f}}$ is obtained by reflecting $N_{f}$ to the bisector. If $\tilde{\alpha}$ is the edge of $N_{\tilde{f}}$ obtained by reflecting the edge $\alpha$ to the bisector, then $\delta_{\tilde{\alpha}}=\delta_{\alpha}, \gamma_{\alpha}=1 / \gamma_{\tilde{\alpha}}$ and estimate (3.30) applies to the quantity $I^{\tilde{\alpha}}(\lambda):=\sum_{\left|j-k \gamma_{\tilde{\alpha}}\right| \leq \tilde{D}} I_{k j}(\lambda, \tilde{f})$. This gives (choosing $\tilde{D}$ large enough)

$$
\left|I^{\alpha}(\lambda)\right| \leq\left|I^{\tilde{\alpha}}(\lambda)\right| \leq C_{\varepsilon} \lambda^{-\delta_{\tilde{\alpha}}+\varepsilon}=C_{\varepsilon} \lambda^{-\delta_{\alpha}+\varepsilon} .
$$

Finally, if $\alpha$ is the principal edge of $N_{f}$, then estimate (3.29) holds assuming the multiplicity condition (3.29). Otherwise the exponent $\frac{A_{\alpha}^{\prime}+\gamma_{\alpha}\left(B_{\alpha}^{\prime}-1\right)-r_{\alpha} \gamma_{\alpha}}{r_{\alpha}+1}-1$ is negative, and choosing $\varepsilon>0$ small enough, one can sum estimate (3.28) in $j$ (also trivially in $k$ ) to obtain

$$
\left|I^{\alpha}(\lambda)\right| \leq C_{\varepsilon} \lambda^{-\frac{1}{r_{\alpha}+1}+\varepsilon} .
$$

In this case one can do the analysis both for $f(x, y)$ and $\tilde{f}(x, y)$. In the latter case $r_{\tilde{\alpha}}$ will be the highest multiplicity of a real root of the function $\partial_{x} f_{\alpha}(x, 1)$. Thus estimate (3.31) holds for $r_{\alpha}$ defined to be the minimum of the highest multiplicity of a nonzero real root of $\partial_{y} f_{\alpha}(1, y)$ and the highest multiplicity of a nonzero real root of $\partial_{x} f_{\alpha}(x, 1)$. This finishes the proof of Theorem 3 . 


\section{REFERENCES}

[AV] V.I. Arnold, A. Gussein-Zade, A. Varchenko: Singularities of differentiable mappings, Vols. I and II, Monographs in Math., vols. 82 and 83, Birkhäuser, Boston, 1985, 1988. MR0777682 (86f:58018), MR0966191 (89g:58024)

[G] A. Greenleaf: Principal curvature and harmonic analysis, Indiana Univ. Math. J. 30 (1981), no. 4, 519-537. MR620265 (84i:42030)

$[\mathrm{H}] \quad$ L. Hörmander: The analysis of linear partial differential operators, I and II, Grundlehren der Mathematischen Wissenschaften, 256 and 257, Springer-Verlag, Berlin (1983). MR0717035 (85g:35002a), MR0705278 (85g:35002b)

[K] V.N. Karpushkin: A theorem on uniform estimates for oscillatory integrals with a phase depending on two variables, Trudy Sem. Petrovsk. No. 10 (1984), 150-169, 258. MR0778884 (86k:58118)

[PS1] D.H. Phong, E.M. Stein, The Newton polyhedron and oscillatory integral operators, Acta Math. 179 (1997) 107-152. MR:1484770 (98j:42009)

[PS2] D.H. Phong, E.M. Stein: Oscillatory integrals with polynomial phases, Invent. Math. 110 (1992) 39-62. MR.1181815 (93k:58215)

[PS3] D.H. Phong, E.M. Stein, J. Sturm: On the growth and stability of real analytic functions, Amer. J. Math. 121 (1999). MR.1738409 (2002a:58025)

[R] V.S. Rychkov: Sharp $L^{2}$ bounds for oscillatory integral operators with $C^{\infty}$ phases, Math. Z. 236 (2001), no. 3, 461-489. MR.1821301 (2002i:42016)

[S] E.M. Stein: Harmonic Analysis: Real-Variable Methods, Orthogonality and Oscillatory Integrals, Princeton University Press (1993). MR1232192 (95c:42002)

[Sh] H. Schulz: On the decay of the Fourier transform of measures on hypersurfaces, generated by radial functions, and related restriction theorems (unpublished).

[T] P. Tomas: A restriction theorem for the Fourier transform, Bull. Amer. Math. Soc. 81 (1975), 477-478. MR0358216 (50:10681)

Department of Mathematics, University of Georgia, Athens, Georgia 30602

Current address: Department of Mathematics, University of British Columbia, 1984 Mathematics Road, Room 121, Vancouver, British Columbia V6T 1Z2, Canada

E-mail address: amagyar2000@yahoo.com 